

\section{DISCLAIMER}

This report was prepared as an account of work sponsored by an agency of the United States Government. Neither the United States Government nor any agency thereof, nor any of their employees, makes any warranty, express or implied, or assumes any legal liability or responsibility for the accuracy, completeness, or usefulness of any information, apparatus, product, or process disclosed, or represents that its use would not infringe privately owned rights. Reference herein to any specific commercial product, process, or service by trade name, trademark, manufacturer, or otherwise does not necessarily constitute or imply its endorsement, recommendation, or favoring by the United States Government or any agency thereof. The views and opinions of authors expressed herein do not necessarily state or reflect those of the United States Government or any agency thereof. 


\section{DISCLAIMER}

Portions of this document may be illegible in electronic image products. Images are produced from the best available original document. 


\title{
X-ray Flashes in ROSAT PSPC Data
}

\author{
X. Sun ${ }^{*}$, H. Li ${ }^{*}$, E. E. Fenimore*, Q. D. Wang ${ }^{\dagger}$ \\ *NIS-2, MS D436, Los Alamos National Laboratory, Los Alamos NM 87545 \\ ${ }^{\dagger}$ Northwestern University, 2131 Sheridan Road, Evanston, IL 60208
}

\begin{abstract}
.
We find 24 short (200-2500s) X-ray flashes from all the ROSAT Position Sensitive Proportional Counter (PSPC) observations above galactic latitude 30 degrees ( $~ 9.6$ $\mathrm{Msec})$ and some observations toward low latitudes $(\sim 2.4 \mathrm{Msec})$. The brightest flash is quite extraordinary. Its flux rises from nondetection by a factor of $>200$ in less than $100 \mathrm{sec}$. Its spectrum can be fitted either by a thermal plasma model with $k T \geq 2.4$ $\mathrm{keV}$, or by a single power-law with the photon number index of $\approx-1.7$. Photon statistics are not sufficient to obtain spectra for other flashes. The flash event fields can vary from optically crowded regions to blank fields. Although eight flashes are found from observations from nearby galaxies, and three of them are within the optical extent of their corresponding galaxies, we could not statistically establish that there are two different populations between nearby galaxies and control fields.
\end{abstract}

\section{INTRODUCTION AND DATA SELECTION}

The sensitivity of ROSAT makes it an excellent choice for searching for X-ray flashes. Partly motivated by the recent discoveries of long X-ray tails that are associated with gamma-ray bursts (GRBs), we have carried out an extensive search for short events in ROSAT/PSPC data. We used all observations longer than 5000s above galactic latitude $30^{\circ}(\sim 9.6 \mathrm{Msec})$, and observations toward bright nearby galaxies at low latitude are also included in the analysis as well as some randomly selected control fields $(\sim 2.4 \mathrm{Msec})$. We excluded the observations toward galaxies closer than $100 \mathrm{kpc}$. The resulting total observing time is about $12 \mathrm{Msec}$.

\section{DATA ANALYSIS}

We perform our searches in the hard band $(0.5-2 \mathrm{keV})$ and the procedure can be summarized as follows:

1) We divide each observation into time segments by $\delta t$. We apply "local", "map", and maximum likelihood source detection methods in combination with background fitting as described in [1] for each segment.

2) A flash candidate is identified when (a) it is detected only in one or two adjacent 
time segments, (b) it has a chance probability of less than $3 \times 10^{-7}$ (Lampton method, [2]), and (c) it has at least 7 photons. These requirements eliminate both the bright sources and the long transient events.

3) These candidates are checked against various instrumental effects, which include, for example, abnormally high background intervals, sources close to the support structure of the PSPC front window, sources inconsistent with the point spread function in either sky or detector coordinates, and times when the satellite pointings were unreliable.

4) We also require that the flash flux is at least a factor of 5 larger than the preor post-flash fluxes. This requirement should exclude most flares from flare stars.

5) We then cross check our candidates with known catalogs, including the MPE, WGA, GSC catalogues, SIMBAD \& NED databases, and DSS images.

The duration of the X-ray flash from GRB, soft gamma-ray repeaters, X-ray bursts, and stellar flares can vary from seconds to $10^{4} \mathrm{~s}$. For short durations, the ROSAT background is negligible and detection is limited by source photon number. Detailed simulations suggest that the optimal search window (at least for finding $\mathrm{X}$-ray flashes associated GRBs) is about $10^{3} \mathrm{~s}$ (Li et al., this Proceedings). We tested our searches using 402, 804 and $1206 \mathrm{~s}$ (ROSAT has a wobbling period of $402 \mathrm{~s}$ ), and found that $1206 \mathrm{~s}$ case does not lose short events in at least $95 \%$ of the cases. The results reported here are based on this time scale.

\section{RESULTS}

Table 1 lists all 24 flash events we have found. The first 8 sources are from directions of nearby galaxies and 3 of those are within the optical extent of their corresponding galaxies. The remaining 16 sources are from control fields. About $1 / 3$ of our events show weak persistent emissions. Since the typical ROSAT point spread function (PSF) is $>30^{\prime \prime}$, counterpart identification is rather difficult. In most of the error boxes, we can find one object (or more) from other waveband. Nevertheless, we tentatively categorize the events into the following groups:

Group 1 has 3 sources, which are within the optical angular extent of nearby galaxies: source \# 2 (M31), 7 and 8 (both in M101). The two M101 sources may indeed be associated with the galaxy because there is no obvious foreground object and the implied flash luminosity is a few times $10^{39} \mathrm{ergs} / \mathrm{s}$. Source \#2 is from the M31 bulge direction and its error box contains some foreground stars.

Group 2 has only one source \# 3, which has no obvious optical (DSS), infrared (IRAS $2560100 \mu \mathrm{m}$,) and radio (VLA NVSS 1.4G) counterparts.

Group 3 is the remaining 20 sources, and their error boxes generally contain at least one object. Some of them contain stars brighter than 10 mag: sources \# 12 (A-type, B=9.1), 13 (G-type, $\mathrm{B}=10.4, \mathrm{~V}=9.4$ ), 5 (A-type, $\mathrm{B}=9.8, \mathrm{~V}=9.3$ ) and 19 (unclassified star, $\mathrm{B}=9.8, \mathrm{~V}=10.3$ ). Other source locations contain optical counterparts fainter than $10 \mathrm{mag}$, including source \# 10 (toward the open cluster Pleiades) and our brightest flash, source \# 16. Though all our flashes events have 
TABLE 1. Flash Events List

\begin{tabular}{|c|c|c|c|c|c|c|c|c|}
\hline $\mathrm{No}^{\mathrm{a}}$ & Obs Seq & $\underset{0}{\text { LII }}$ & BII & $\begin{array}{l}\text { Start Time of flash } \\
\text { dd-mm-yy hh:mm }\end{array}$ & $\begin{array}{c}T_{d u r} \\
\text { sec }\end{array}$ & $\begin{array}{r}F_{\text {flash }} \\
10^{-3} \mathrm{c} / \mathrm{s}\end{array}$ & $\begin{array}{l}F_{p r e} \\
10^{-3} \mathrm{c} / \mathrm{s}\end{array}$ & $\begin{array}{l}F_{\text {post }} \\
10^{-3} \mathrm{c} / \mathrm{s}\end{array}$ \\
\hline $1^{*}$ & 600304p & 120.670 & -21.606 & $31-12-92$ 05:59 & 1500 & 8.8 & $<2$ & $<1.6$ \\
\hline $2^{* \dagger}$ & $600064 p$ & 120.907 & -21.581 & $16-07-9106: 32$ & 600 & 78 & $\sim 2$ & $\sim 3$ \\
\hline 3 & $701183 p$ & 261.824 & -53.285 & $22-12-9221: 33$ & 1060 & 17 & - & $<1$ \\
\hline 4 & $600145 p$ & 140.323 & 43.678 & $10-11-9103: 36$ & 1380 & 4.8 & $<0.6$ & - \\
\hline $5^{*}$ & $700012 p$ & 309.661 & 18.842 & $03-02-9220: 52$ & 1020 & 30 & $<3$ & $<13$ \\
\hline $6^{*}$ & $600188 \mathrm{p}$ & 315.033 & 32.345 & 09-01-93 12:35 & 1800 & 30 & $<5$ & $\sim 3$ \\
\hline $7^{* \dagger}$ & $600108 p$ & 102.189 & 59.876 & $08-06-9120: 37$ & 2430 & 8.3 & $\sim 1.2$ & $<2.6$ \\
\hline $8^{\dagger}$ & $600108 p$ & 102.116 & 59.724 & $08-06-9120: 58$ & 800 & 10 & $<1$ & $<2$ \\
\hline 9 & $700101 p$ & 109.436 & -41.124 & $30-06-91 \quad 17: 19$ & 1450 & 4.4 & $<0.6$ & $<5$ \\
\hline 10 & $200068 \mathrm{p}$ & 166.882 & -23.930 & $23-08-9103: 58$ & 2160 & 14 & $\sim 1.3$ & $\sim 1.1$ \\
\hline 11 & $800278 p$ & 219.643 & -36.347 & $03-09-9221: 54$ & 1950 & 8.1 & $<1.4$ & $<4$ \\
\hline 12 & $200547 \mathrm{p}$ & 170.692 & -5.555 & 05-09-91 06:55 & 620 & 35 & $<2$ & $<1$ \\
\hline $13^{*}$ & $201382 \mathrm{p}$ & 162.164 & 41.555 & $29-10-92 \quad 13: 20$ & 930 & 20 & $<2$ & $\sim 2.2$ \\
\hline $14^{*}$ & $200956 p$ & 212.848 & 58.727 & $11-05-93 \quad 04: 48$ & 1400 & 21 & - & $<1$ \\
\hline 15 & $800410 p$ & 179.315 & 60.219 & $01-12-92 \quad 04: 34$ & 700 & 11 & $<2$ & $\sim 1.7$ \\
\hline 16 & $150071 p$ & 31.739 & 70.626 & $23-07-9008: 35$ & 810 & 260 & $<1$ & $\sim 5.6$ \\
\hline $17^{*}$ & $800385 p$ & 6.490 & 30.561 & $01-09-9222: 53$ & 900 & 15 & 一 & $<1$ \\
\hline 18 & $800281 p$ & 21.338 & 33.279 & $05-09-92 \quad 16: 07$ & 870 & 21 & $<2$ & $<3$ \\
\hline 19 & $200150 p$ & 344.379 & 1.805 & $02-03-9105: 10$ & 360 & 38 & $<4$ & $<1$ \\
\hline 20 & $400151 p$ & 356.931 & -4.544 & $23-09-9104: 33$ & 930 & 23 & $<4$ & $<67$ \\
\hline $21^{*}$ & $400146 p$ & 1.567 & -11.966 & $03-04-92 \quad 19: 55$ & 840 & 44 & $<4$ & $<10$ \\
\hline $22^{*}$ & $900403 p$ & 32.251 & -0.234 & 05-04-93 12:02 & 690 & 110 & $<1$ & $<2$ \\
\hline $23^{*}$ & $300121 p$ & 70.427 & -30.050 & $11-05-92 \quad 07: 45$ & 1620 & 6.3 & 一 & $<1$ \\
\hline 24 & $800488 p$ & 93.754 & -38.382 & $09-12-9309: 33$ & 270 & 37 & $<1$ & $\sim 7.9$ \\
\hline
\end{tabular}

${ }^{a}$ * indicates the source also included in either the MPE and/or WGA catalogue. $\uparrow$ indicates it is within the optical extent of a nearby galaxy.

src\#2: In M31 bulge direction. There are a number of stars listed in the BHG88 catalogue within the X-ray error circle. Three emission line objects are also within the error circle. src\#4: Only one uncatalogued optical object is within the error circle in the DSS image. src\#5: An A type star HD116810 (B=9.8, V=9.3) in the error circle.

src\# 7: Error circle includes several HII regions and SNRs that are in M101.

src\# 8: Error circle is centered between two north arms of M101, several HII regions and SNRs that are from M101 are at the edge of error circle.

src\#9: Flash from a blank field of optical (DSS), infrared (IRAS 25, 60,100 $\mu \mathrm{m}$ ) and radio (VLA NVSS $1.4 \mathrm{GHz}$ ).

src\# 10: In direction of the Pleiades star cluster. Error circle includes Melotte $22 \mathrm{HHJ} 104$ $(\mathrm{V}=18.9)$.

src\# 12: Error circle includes an A2 star, HD 282674, (B=9.1).

src\#13: Error circle includes a G-type star $A G+55(B=10.4, V=9.4)$.

src\#15: Error circle includes an IRAS F10382+4005 and a small diffuse optical object.

src\#16: The brightest flash in our events.

src\#19: Error circle includes a bright star CPD-40 $7586(\mathrm{~B}=9.8, \mathrm{~V}=10.3$.

src\#24: Flash has fluxes in both soft and hard band. 

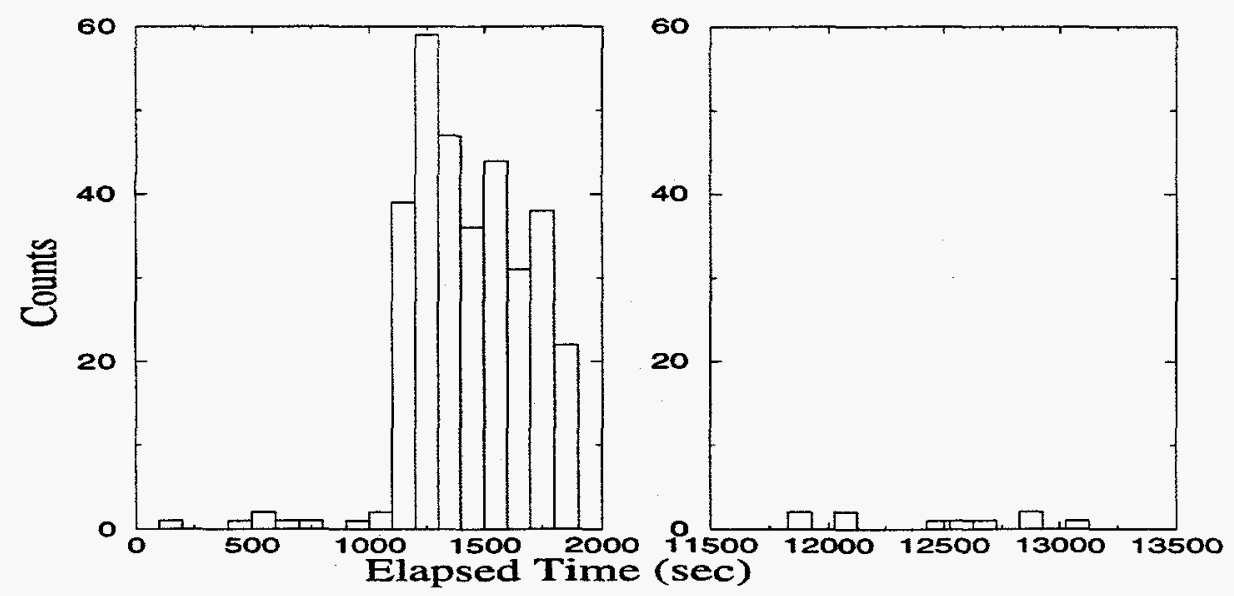

FIGURE 1. Broad band lightcurve of our brightest flash (source \# 16). The time history is binned at $100 \mathrm{sec}$. This flash has a rise time $\sim 100 \mathrm{sec}$ with a flux jump from nondetection by a factor of 260. Unfortunately, it is interrupted by a data gap for $\sim 10^{4} \mathrm{sec}$ and when it reappears, its flux is down by a factor of 46 . There is $\sim 14,000$ sec observation before the flash from which we only found an upper limit.

large ( $\geq 5$ ) flux variations, it is conceivable that some of those flashes may actually be flares from those stars. Since most of our events are weak, we cannot do any spectral fitting to see whether their spectra are consistent with the ordinary flares.

\section{DISCUSSION AND CONCLUSION}

It is difficult to compare our findings with previous work since the instrument sensitivities, energy bands, search techniques are quite different. Connors et al. [3] found 8 events with peak fluxes of $10^{-10}$ to $-9 \mathrm{erg} / \mathrm{cm}^{2} / \mathrm{s}$ (2-10keV) using HEAO1 A2. Gotthelf et al. [4] used the Einstein IPC (which is similar to the ROSAT PSPC) and found 42 events down to $10^{-11} \mathrm{erg} / \mathrm{cm}^{2} / \mathrm{s}(0.1-4.0 \mathrm{keV})$. But they only searched for very short events, $\sim 10 \mathrm{~s}$. From the complete ROSAT PSPC data at high galactic latitude $\left(\geq 30^{\circ}\right)$ and some of the PSPC data at low latitude, we found 24 flash-like events (200-2500s) down to $5 \times 10^{-13} \mathrm{erg} / \mathrm{cm}^{2} / \mathrm{s}(0.5-2.4 \mathrm{keV})$.

Two of our flashes have A stars brighter than 10 mag in their error boxes, but it is unclear whether those are single or binary systems. Simon et al. [4] surveyed the X-ray emission in direction of $74 \mathrm{~A}$-type stars and found that nine supposedly single late-A stars are coincident with X-ray sources. We do not yet have a good physical understanding of how single A stars make X-ray emissions/flares or if the $\mathrm{x}$-rays are actually all coming from the unseen late-type companions.

The brightest flash event we found is source \# 16, and Figure 1 shows its lightcurve. This source is very interesting in both its temporal profile and spectrum. It has emissions in both the soft and hard bands. Only an upper limit is obtained in the pre-flash interval and it is $\sim 260$ times lower than the flux during 
TABLE 2. Spectral Fits of the brightest source \# 16

\begin{tabular}{ccccc} 
Model & $\begin{array}{c}\text { Continuum } \\
\Gamma \text { or } k T(\mathrm{keV})\end{array}$ & $\begin{array}{c}N_{H} \\
\left(\times 10^{20}\right)\end{array}$ & $\begin{array}{c}\text { Flux } \\
\left(\mathrm{erg} \mathrm{cm}^{-2} \mathrm{~s}^{-1}\right)\end{array}$ & $\chi_{\nu}^{2}(\nu)$ \\
\hline Power Law & $-1.7 \pm 0.3$ & $1.8 \pm 0.8$ & $6.5 \times 10^{-12}$ & $0.58(10)$ \\
Thermal (R-S) & $2.4_{-0.7}^{+\infty} \mathrm{keV}$ & $1.4 \pm 0.6$ & $5.7 \times 10^{-12}$ & $0.47(10)$ \\
\hline
\end{tabular}

the flash, while only the hard band emission was detected during the post-flash interval and it is $\sim 46$ times lower than that during the flash. The true duration is not known since there is an orbit gap $\left(\sim 10^{4}\right)$ and when the source reappears, its flux is already down by a factor of 46 , hardly detectable. There is evidence of spectral hardening during the flash. Table 2 lists the spectral fits we have obtained. The thermal plasma temperature quoted is only the lower bound due to the low energy coverage of ROSAT. Furthermore, a single temperature black body does not give an acceptable fit (the reduced $\chi_{\nu}^{2}(\nu) / d o f=3.9$, where $\nu=10$ is the degrees of freedom). The Galactic $N_{H}$ value in this direction is $1.6 \times 10^{20} \mathrm{~cm}^{-2}$.

There is, however, one faint optical object in DSS image and its color is red (D. Lamb, private communication), which probably rules out an AGN. In addition, an amplitude variation by a factor of 260 in less than a few minutes has probably never been seen from an AGN. If the power-law fit represents the true source spectrum, then it is difficult to categorize this source since it does not seem to fit with many known types of transients from flare stars, RS CVns, X-ray bursters, etc.

On the other hand, if the thermal plasma (Raymond-Smith model) fit with $k T \geq$ $2.4 \mathrm{keV}$ is the correct description, then this flash could be a super flare from an $\mathrm{M}$ dwarf flare star or a huge flare from an active binary such as RS CVn and Algoltype systems (see [6], [7], [8]). Flares from RS CVns typically have slightly higher temperatures $(\sim 7 \mathrm{keV})$ than that in $\mathrm{M}$ dwarf flares $(\sim 2-3 \mathrm{keV})$, and tend to be much longer $\left(\sim 10^{5} \mathrm{sec}\right)$. The source luminosity is $\sim 8 \times 10^{30} d_{100}^{2} \mathrm{ergs} / \mathrm{s}$, where $d_{100}$ is distance in $100 \mathrm{pc}$ and it generates $\sim 10^{34} d_{100}^{2}$ ergs in the first $1000 \mathrm{sec}$. Since both the best fit plasma temperature and the implied total energy are within the observed ranges of flares from both type of systems, further spectroscopic studies of this source is needed to distinguish between these two systems.

We acknowledge enlightening discussions with Don Lamb.

\section{REFERENCES}

1. Zimmermann, H.U., et al., EXSAS User's Guide, MPE Rep. 257 (1994).

2. Theiler, J.P., Ap. J., to be submitted, on Lampton method for source detection.

3. Connors, A., Serlemitsos, P.J. \& Swank, J.H., Ap.J., 303, 769 (1986).

4. Gotthelf, E.V., Hamilton, T.T \& Helfand, D.J., Ap.J., 466, 779 (1996).

5. Simon, T., Drake, S. \& Kim, P., Pub. Astr. Soc. Pacific, 107, 1034 (1995).

6. Pallavicini, R., Flares and Flashes, Springer-Verlag, Berlin, 1995, pp. 148.

7. Stern, R.A., and Underwood, J.H., Ap. J., 264, L55 (1983)

8. Ottmann, R., Flares and Flashes, Springer-Verlag, Berlin, 1995, pp. 164 